\section{TUMOUR IMMUNOLOGY}

\section{Stalemate}

It has been proposed that the immune system can maintain tumours in a state of equilibrium, such that the tumour does not regress or expand. Clear evidence for this state of play has now been produced by Mark Smyth, Robert Schreiber and colleagues.

The immune system has crucial roles in shaping cancer development: it is able to detect early lesions through immunosurveillance and eliminate them; it is thought to maintain tumours in a state of equilibrium; and tumour cells with either reduced immunogenicity or with increased

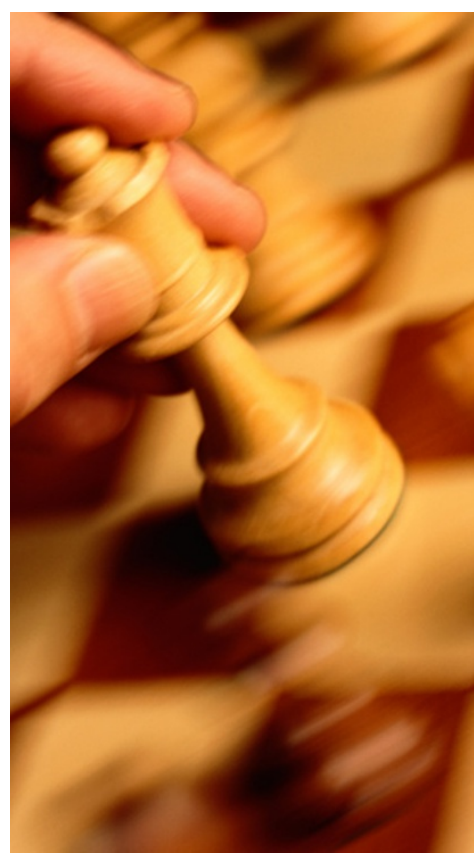

capacity to attenuate the immune response can escape equilibrium and progress. This whole process is termed cancer immunoediting.

The authors used C57BL/6 and $129 / \mathrm{SvEv}$ mouse colonies established in two different laboratories and injected age and sex matched groups with 3'-methylcholanthrene (MCA), a chemical carcinogen known to induce sarcoma formation in mice. As the authors were only interested in the mice that did not develop progressively growing tumours, they eliminated from the study all mice that had expanding tumours by 200 days. Mice that had only small stable masses at the injection site were then treated with either control immunoglobulins or a mixture of monoclonal antibodies to deplete $\mathrm{CD} 4^{+}$and $\mathrm{CD} 8^{+}$ $\mathrm{T}$ cells and to neutralize interferon- $\gamma$ (IFN $\gamma$ ). $60 \%$ of these mice developed progressively growing tumours whereas no tumours were seen in the control mice. These experiments were repeated several times with similar results. Importantly, suppression of natural-killer-cell function did not induce tumour outgrowth, indicating that suppression of the adaptive immune response is influential.

To examine this process more carefully, the authors used mice deficient in recombination-activating

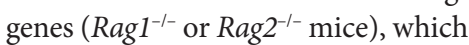
have an innate, but not an adaptive immune response. When subjected to the same MCA and monoclonal antibody protocol, very few of these mice developed late-onset tumours; most developed progressively expanding tumours by 200 days. In the mice that did develop late-onset tumours, the time to tumour outgrowth was prolonged compared with that of the tumours that grew out in wild-type mice treated with antibodies against CD4, CD8 and IFN $\gamma$ at 200 days. The more rapid outgrowth of tumours in the wild-type immunosuppressed mice indicate that the tumours probably have fully transformed cells that can proliferate in the absence of the adaptive immune response. This was further supported by the fact that atypical fibroblast-like cells that grew out of the stable MCA-generated masses from the wild-type mice formed tumours when injected into Rag2 $^{-/-}$mice.

Interestingly, some of the control wild-type mice developed late-onset sarcomas, indicating that these might arise from cells that had escaped equilibrium. In agreement with cancer immunoediting, cells explanted from these tumours should be able to evade the immune response in syngeneic mice, unlike those that exist in the small masses, which should be immunogenic. The authors confirmed this with most mice developing tumours when injected with cells from the lateonset sarcomas, whereas up to $51 \%$ of the cells from the small masses were rejected.

These results clearly indicate that the immune system can maintain tumours in a state of equilibrium and that cells that escape this process are more able to evade the immune response.

Nicola McCarthy, Senior editor, Nature Reviews Cancer

ORIGINAL RESEARCH PAPER Koebel, C. M. et al. Adaptive immunity maintains occult cancer in an equilibrium state. Nature 18 November 2007 (doi:10.1038/nature06309) 